\title{
Analysis of the prevalence of allergens listed in European baseline series in products composition used in atopic dermatitis available in Polish online drugstores
}

\author{
Katarzyna Osinka*, Agnieszka Mysliwiec, Wojciech Feleszko, Agnieszka Krauze
}

From EAACI Skin Allergy Meeting 2014

Krakow, Poland. 18-20 September 2014

\section{Background}

Atopic dermatitis (AD) is a chronic, recurrent inflammatory skin disease. Due to deficient skin barrier function, patients with $\mathrm{AD}$ are exposed to higher risk of contact sensitization and allergic contact dermatitis. Basic therapy of AD should consist of optimal skin care pointed at the skin barrier defect with regular use of emollients. Unfortunately, products from this group can potentially comprise compounds listed in European Baseline Series (EBS) used in the patch test allergens. The aim of this study was to determine the prevalence of allergens listed in European Baseline Series included in preparations available in polish online drugstores.

\section{Method}

About 40 online drugstores were searched by two independent researchers. We included preparations described by the manufacturers as "emollients" or "products able for treatment of atopic dermatitis" and subsequently assessed for their presence of chemical compounds included to list of the EBS(28 most common allergens). The analysis also included the cost of each product (all collected on the same day, 28 Jan 2014).

\section{Results}

196 preparations met our inclusion criteria. 17 of them $(8,7 \%)$ weren't examined because of the lack of information about ingredients based on INCI (International Nomenclature of Cosmetics Ingredients). The analysis of the data revealed that $60(58,3 \%)$ emollient products

Medical University of Warsaw, Pediatric Pulmonology and Allergology Clinic, Warsaw, Poland 